\title{
Automatic Coin Recogniton Using Local Spatial Features
}

\author{
Unnikrishnan G, Sajith sethu P \\ (PG Scholar, SCT College of Engineering, Trivandrum, Kerala, India \\ (Assistant Professor, SCT College of Engineering, Trivandrum, Kerala, India
}

\begin{abstract}
Coins are integral part of our day to day life and now it becomes a basic need that coins can be sorted and counted automatically. This paper describes Automated Coin Recognition System for the recognition of Indian Coins with translation and rotation invariance. The paper presents a novel approach that uses the normalized local spatial features of the coin image to derive an abstract image. Decomposition of abstract image in to concentric circles is then used to extract a set of compact and effective features. The proposed method uses a single gallery image per coin for the recognition and show a robust and high recognition performance of $97.43 \%$ under variable conditions such as rotation and translation.
\end{abstract}

Keywords: Image abstraction, Hough transform

\section{INTRODUCTION}

The recent explosion of digital image resulted in most advance image processing techniques for coin recognition. This includes the statistical and structural approaches. The statistical approach deals with statistical characterization of the coin image. On the other hand the structural method is based on the structural relationship between the features from the coin image. Query by Visual Example (QVE) [3], focuses on the problem of image matching. This method uses block correlation scheme for comparison and is not invariant to translation. Edge histogram descriptor (EHD) [4], an enhanced and more efficient version of the approach consists of a 150-bin histogram. A given image is divided into 16 sub-images first, local edge histograms are then computed for each sub-image based on the strength of five different edges. The Dagobert system has been developed for coin recognition and sorting [5]. The system relays on a correlation method based on canny edge operator. Coin image recognition is based on estimating a rotation angle and it is used to align the coin under process to master coins. These are some of the important image processing techniques used for recognizing the coins.

The presented paper is a novel approach which divides the abstract images in to number of concentric circles. The feature extracted for the matching process the concatenated histogram of all the concentric circles. The abstract image is derived from the spatial intensity changes in the images. Normalized Local standard deviation filter is used for extracting the spatial intensity changes in the image. The key feature of this approach is that it uses single gallery image per coin for the recognition purpose and produces high recognition accuracy. This also reduces the database size of gallery images into considerable amount. Using background extraction of the coin and the decomposition of image into concentric circles increases its invariant property against rotation and translation.

\subsection{Extract coin from its background}

\section{Implementation details}

As all the Indian coins have the shape that of a circle, circular Hough transform which detects the circular shape on the image is used for extracting the coin from its background. This extracted coin is further used for processing. The general Hough transform can be described as a transformation of a point in the $\mathrm{N}$ dimensional plane to the parameter space. The parameter space is defined according to the space of the object of interest. The equation of the circle is

$$
r^{2}=(x-x 1)^{2}+(y-y 1)^{2}
$$

Where $x 1$ and $y 1$ are the centers of the circle and $r$ is the radius. The parameter representation of circle is

Algorithm

$$
\begin{aligned}
& x=x 1+r \cos (\theta) \\
& x=x 1+r \sin (\theta)
\end{aligned}
$$

1. Define a 3-dimensional Hough Matrix of $(\mathrm{K} \times \mathrm{L} \times \mathrm{R})$, where $\mathrm{K}, \mathrm{L}$ is the height and width of the Gray scale image and $\mathrm{R}$ is the number of radii for which we want to search.

2. For each edge pixel $(\mathrm{x}, \mathrm{y})$ and for particular radius $\mathrm{r}$, search circle center coordinates $(\mathrm{x} 1, \mathrm{y} 1)$ that satisfy the equation $r^{2}=(x-x 1)^{2}+(y-y 1)^{2}$ and increase count in Hough Matrix at $(x 1, y 1, r)$ by 1 . 
3. Repeat step 2 for other radii.

4 Find the maximum value from the Hough Matrix. The corresponding indices give the center coordinates and radius of coin.

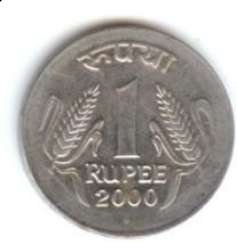

Fig 1 Original image having White background and shadow

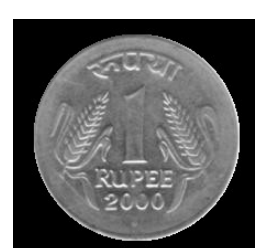

Fig 2 Coin after background and shadow removal

Since only the coin segmented from the background and is processed for further image abstraction and decomposition it is very much invariant to translation. This also removes if there is any shadow occurred while taking the image and hence considerably increases the performance of the recognition system.

\subsection{Image abstraction}

An abstract image is obtained by the following approach. The color image is first converted to its gray image. Spatial intensity change in the image is the essential feature required for recognition. There are many ways of calculating the spatial intensity changes from the raw vector. Gradient and range are some of the filters. In the proposed method as a novel procedure the spatial change intensity is calculated using the local standard deviation filter on each of the pixel by applying the equation

$$
\sigma=\sqrt{\frac{1}{m n} \sum_{s=-a}^{a} \sum_{t=-b}^{b}[x(i+z, j+t)-\bar{x}(i, j)]^{2}}
$$

Where $\mathrm{a}=(\mathrm{m}-1) / 2$ and $\mathrm{b}=(\mathrm{n}-1) / 2$. The value of $\bar{x}(i, j)$ is given by

$$
\bar{x}(i, j)=\frac{1}{m n} \sum_{s=-a}^{a} \sum_{t=-b}^{b} x(i+s, j+t)
$$

To get the feature vector, normalization is performed by dividing each element of $\sigma$ by diving by its region mean $\bar{\sigma}$. Now each pixel of the image has the values within the range 0 and 1 and hence the histogram within this specified range increases the performance. The abstracted image by using the above technique completely encompasses all the features coin required for recognition. The accuracy is calculated with and without the above method and accuracy increases from 20 to $97.43 \%$ by including the above proposed method.

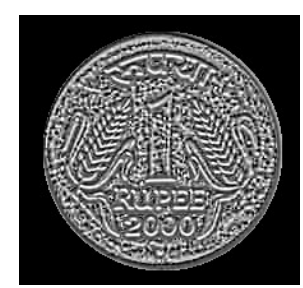

Fig 3 Abstracted image

\subsection{Decomposition of abstract image}

The abstracted image is divided in to $\mathrm{N}$ circular strips having the center as obtained from the Hough matrix for coin extraction. Each circular strip is having the width r. A typical strip ci has the boundary concentric circles with radii $i r$ and $(i+1) r$ for $\mathrm{i}=2,3,4 \ldots \ldots . . \mathrm{N}$. For $\mathrm{i}=1$ we define a circle of radius $\mathrm{r}$.

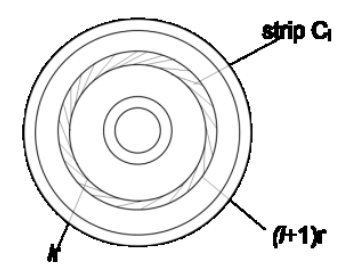

Fig 4 Decomposed image 
The horizontal concatenation of histogram of each concentric circle is the feature vector of the coin image. The features extracted above are rotation invariant because the histogram of any circular strip remains in the same strip under any degree of rotation. The strip width is a discretionary variable which can be used for fine-tocoarse selection of the extracted features.

The similarity is measured using the mean absolute difference classifier. The test image is matched with the trained images and the image in the test database corresponding to the minimum match is the recognized coin.

\section{Experimental Results}

The Indian coins of denominations 1,2,5 and 10 are scanned from both sides. The denominations 1,2 and 5 have two types' coins. So a total of 14 images are scanned. The database for the test images is created by rotating the scanned images at different degrees and translating the images at different pixel shifts in all direction. Images were rotated at $10^{\circ}, 20^{\circ}, \ldots \ldots . .360^{\circ}$, that is a total of 36 images. For translation the image is shifted in pixel wise 5 times in X-direction, Y-direction and diagonally to get a total of 15 images. So the test database has a total of 700 images. The train image is the neutral image without shift or rotation. That is the train database consists of only 14 images. Mean absolute error classifier is used for similarity measurement. Each test image is compared with all the train images and the mean absolute error is calculated. The train image corresponding to min absolute error is the recognized image. The procedure is done for all the test images and the recognition accuracy is measured. The proposed method produces an accuracy of $97.43 \%$. The proposed method is also validated with features without the spatial change intensity change. Here the accuracy reduces to $97.5 \%$ to $20 \%$, thus shows the importance of using the spatial intensity change features.

\begin{tabular}{|c|c|c|c|}
\hline Sr No & Coin Type & $\begin{array}{c}\text { Images correctly } \\
\text { recognized/total } \\
\text { images }\end{array}$ & Accuracy \\
\hline 1 & $`$ & $196 / 200$ & $98 \%$ \\
\hline 2 & $`$ & $194 / 200$ & $97 \%$ \\
\hline 3 & $` 5$ & $192 / 200$ & $96 \%$ \\
\hline 4 & $` 10$ & $100 / 100$ & $100 \%$ \\
\hline 5 & Total & $682 / 700$ & $97.43 \%$ \\
\hline
\end{tabular}

Table 1 Recognition Results

\section{Conclusion}

The proposed method uses the local features of the image for feature extraction. The method is invariant to rotation and translation and also the recognition is being done with single neutral image as train image. Even with this less number of train images an accuracy of $97.43 \%$ is being met. By adding more spatial features the accuracy can be increased hence the proposed method can be used as an efficient method for the face recognition system also.

\section{Reference}

[1]. R. Bremananth, B. Balaji, M. Sankari, and A. Chitra, .A new approach to coin recognition using neural pattern analysis,. in Proc. IEEE Annual INDICON, 2005, pp. 366.370.

[2]. M. Fukumi, S. Omatu, and Y. Nishikawa, .Rotationinvariant neural pattern recognition system estimating a rotation angle,. IEEE Transactions on Neural Networks, vol. 8, no. 3, pp. 568.581, 1997.

[3]. K. Hirata and T. Kato, Query by visual examplecontent based image retrieval,. in Advances in Database Technology - EDBT '92, Berlin, Germany, 1992, pp. 56.71.

[4]. ISO/IEC JTC1/SC29/WG11/N4063, .MPEG-7 Visual part of eXperimentation Model Version 10.0, Singapore, March 2001.

[5]. M. N"olle, P. Harald, R. Michael, K. Mayer,I. Holl"ander, and R. Granec, .Dagobert - a new coin recognition and sorting system,. in Proc. DICT A Digital image computing techniques and applications, 2003, vol. 1, pp. 329.338

[6]. Cai-ming Chen, Shi-qing Zhang, Yue-fen Chen, "A Coin Recognition System with Rotation Invariance," 2010 International Conference on Machine Vision and Humanmachine Interface, 2010, pp. 755-757.

[7]. Shen, L., Jia, S., Ji, Z. and Chen, W.S., "Statictics of Gabor features for coin reco gnition", IEEE International Workshop on Imaging Systems and Techniques, 2009, pp. 295 - 298.

[8]. Gupta, V., Puri, R., Verma, M., "Prompt Indian Coin Recognition with Rotation Invariance using Image

[9]. Subtraction Technique", International Conference on Devices and Communications (ICDeCom), 2011 